\title{
Necessary and sufficient condition for existence of periodic solutions of predator-prey dynamic systems with Beddington-DeAngelis-type functional response
}

\author{
Neslihan Nesliye Pelen ${ }^{1 *}$, A. Feza Güvenilir ${ }^{2}$ and Billur Kaymakçalan ${ }^{3}$
}

\author{
${ }^{*}$ Correspondence: \\ nesliyeaykir@gmail.com \\ 'Department of Mathematics, \\ Ondokuz Mayıs University, Samsun, \\ Turkey \\ Full list of author information is \\ available at the end of the article
}

\begin{abstract}
We consider two-dimensional predator-prey systems with Beddington-DeAngelis-type functional response on periodic time scales. For this special case, we try to find the necessary and sufficient conditions for the considered system when it has at least one $w$-periodic solution. This study is mainly based on continuation theorem in coincidence degree theory and will also give beneficial results for continuous and discrete cases. Especially, for the continuous case, by using the study of Cui and Takeuchi (J. Math. Anal. Appl. 317:464-474, 2006), to obtain the globally attractive $w$-periodic solution of the given system, an inequality is given as a necessary and sufficient condition. Additionally, for the continuous case in this study, the open problem given in the discussion part of the study of Fan and Kuang (J. Math. Anal. Appl. 295:15-39, 2004) is solved.
\end{abstract}

Keywords: predator-prey dynamic systems; Beddington-DeAngelis-type functional response; continuation theorem; globally attractive solution; periodic solution; time-scale calculus

\section{Introduction}

The relationships between species and the outer environment and the connections between different species are the description of the predator-prey dynamic systems, which are the subject of mathematical ecology in biomathematics. Various types of functional responses in a predator-prey dynamic system such as Monod-type, semi-ratio-dependent, and Holling-type have been studied in [3-5].

The key concepts in this study are the functional response in the periodic environment and the time-scale calculus.

First of all, we investigate the predator-prey system with Beddington-DeAngelis-type functional response for a general time scale and its continuous case. This type of functional response first appeared in [6] and [7]. At low densities, with this type of functional response, some of the singular behaviors of ratio-dependent models are avoided. Also,

(c) 2016 Pelen et al. This article is distributed under the terms of the Creative Commons Attribution 4.0 International License (http://creativecommons.org/licenses/by/4.0/), which permits unrestricted use, distribution, and reproduction in any medium, provided you give appropriate credit to the original author(s) and the source, provide a link to the Creative Commons license, and indicate if changes were made. 
predator feeding can be described much better over a range of predator-prey abundances by using Beddington-DeAngelis-type functional response.

Secondly, being in a periodic environment is important because, in such an environment, the global existence and stability of a positive periodic solution is a significant problem in population growth model. This plays a similar role as a globally stable equilibrium in an autonomous model. Therefore, it is important to consider under which conditions the resulting periodic nonautonomous system would have a positive periodic solution that is globally asymptotically stable, and the globally asymptotically stable periodic solution of the given system in the continuous case is investigated in this study as an application. For nonautonomous case, there are many studies on the existence of periodic solutions of predator-prey systems in continuous and discrete models based on the coincidence theory such as [5, 8-16].

Additionally, for the continuous case, the studies of Fan and Kuang [2] and Cui and Takeuchi [1] have very important contributions on the predator-prey dynamic systems with Beddington-DeAngelis-type functional response. They have investigated the following equation:

$$
\begin{aligned}
& \tilde{x}^{\prime}(t)=a(t) \tilde{x}(t)-b(t) \tilde{x}^{2}(t)-\frac{c(t) \tilde{y}(t) \tilde{x}(t)}{\alpha(t)+\beta(t) \tilde{x}(t)+m(t) \tilde{y}(t)}, \\
& \tilde{y}^{\prime}(t)=-d(t) \tilde{y}(t)+\frac{f(t) \tilde{x}(t) \tilde{y}(t)}{\alpha(t)+\beta(t) \tilde{x}(t)+m(t) \tilde{y}(t)} .
\end{aligned}
$$

Here $\tilde{x}$ and $\tilde{y}$ represent the densities of the populations of the prey and predator. In other words, they represent the numbers of individuals in the prey and predator population per unit area, respectively. For general nonautonomous case, Fan and Kuang studied the permanence, extinction, and global asymptotic stability of the given system. For the periodic case, Fan and Kuang established two sufficient criteria for the existence of a positive periodic solution by using Brouwer fixed point theorem and continuation theorem in coincidence degree theory, respectively. These criteria are easy to be verified for the given system in the form of (1). At the same time, authors pointed that these criteria have room for further improvement. They presented numerical simulation to indicate that (1) may admit positive periodic solutions when the conditions in the theorems fail.

On the basis of these obtained results for system (1) with periodic coefficients, Cui and Takeuchi continue the study on the periodic solution and permanence of that system. Cui and Takeuchi obtained some new conditions for the permanence and existence of a positive periodic solution of system (1). These results improve those obtained by Fan and Kuang [2]. In addition to this improvement, in their paper [1], they also give the equivalence between the permanence and satisfaction of the inequality in Theorem 2.1 in [1]. However, they could not show whether there is the equivalence between the existence of at least one $w$-periodic solution and satisfaction of the inequality in Theorem 2.1 in [1].

In this study, for the continuous case, this equivalence is shown by using coincidence degree theory as an application of general time-scale case. In addition to that, we also show the global attractivity or global asymptotic stability of this $w$-periodic solution. In other words, the contribution of this paper is that we prove that any predator-prey dynamic systems with Beddington-DeAngelis-type functional response satisfying the inequality in Theorem 2.1 in paper [1] have the same meaning with having globally attractive $w$-periodic 
solution. Therefore, we are able to show that to obtain a periodic solution for the periodic case of system (1), the improvement of this inequality becomes impossible.

Thirdly, the unification of continuous and discrete analysis is also significant for this study. To unify the study of differential and difference equations, the theory of time-scale calculus is initiated by Stephan Hilger [17]. In $[4,18]$ unification of the existence of periodic solutions of population models modeled by ordinary differential equations and their discrete analogues in the form of difference equations and extension of these results to more general time scales is studied. The aim of this study is to find a necessary and sufficient condition for the periodic solution of the given system with Beddington-DeAngelis-type functional response for a general time scale and apply this result to the continuous case.

In this paper, we investigate the system

$$
\begin{aligned}
& x^{\Delta}(t)=a(t)-b(t) \exp (x(t))-\frac{c(t) \exp (y(t))}{\alpha(t)+\beta(t) \exp (x(t))+m(t) \exp (y(t))}, \\
& y^{\Delta}(t)=-d(t)+\frac{f(t) \exp (x(t))}{\alpha(t)+\beta(t) \exp (x(t))+m(t) \exp (y(t))} .
\end{aligned}
$$

Here $\mathbb{T}$ is periodic, that is, if $t \in \mathbb{T}$ then $t+w \in \mathbb{T}$, and $a(t), b(t), c(t), d(t), f(t), \alpha(t), \beta(t), m(t)$ are $w$-periodic functions in $\mathbb{T}$. We define $w$-periodic functions $h(t)$ in $\mathbb{T}$ by $h(t+w)=h(t)$. All the coefficient functions are positive. This system on time scales was studied in $[4$, $18-20]$.

\section{Preliminaries}

The necessary information is taken from [21]. Let $X, Z$ be normed vector spaces, $L$ : $\operatorname{Dom} L \subset X \rightarrow Z$ be a linear mapping, and $N: X \rightarrow Z$ be a continuous mapping. The mapping $L$ will is called a Fredholm mapping of index zero if $\operatorname{dim} \operatorname{Ker} L=\operatorname{codim} \operatorname{Im} L<+\infty$ and $\operatorname{Im} L$ is closed in $Z$. If $L$ is a Fredholm mapping of index zero and there exist continuous projections $P: X \rightarrow X$ and $Q: Z \rightarrow Z$ such that $\operatorname{Im} P=\operatorname{Ker} L$ and $\operatorname{Im} L=\operatorname{Ker} Q=\operatorname{Im}(I-Q)$, then it follows that $\left.L\right|_{\operatorname{Dom} L \cap \operatorname{Ker} P}:(I-P) X \rightarrow \operatorname{Im} L$ is invertible. We denote the inverse of that map by $K_{P}$. If $\Omega$ is an open bounded subset of $X$, then the mapping $N$ is called $L$ compact on $\Omega$ if $Q N(\Omega)$ is bounded and $K_{P}(I-Q) N: \Omega \rightarrow X$ is compact. Since $\operatorname{Im} Q$ is isomorphic to $\operatorname{Ker} L$, there exists an isomorphism $J: \operatorname{Im} Q \rightarrow \operatorname{Ker} L$.

Definition 1 ([22]) The codimension (or quotient or factor dimension) of a subspace $L$ of a vector space $V$ is the dimension of the quotient space $V / L$; it is denoted by $\operatorname{codim}_{V} L$ or simply by $\operatorname{codim} L$ and is equal to the dimension of the orthogonal complement of $L$ in $V$, and we have $\operatorname{dim} L+\operatorname{codim} L=\operatorname{dim} V$.

The given information is necessary for the following continuation theorem.

Theorem 1 ([23], continuation theorem) Let L be a Fredholm mapping of index zero, and $N$ be L-compact on $\Omega$. Let the following conditions be satisfied:

(a) For each $\lambda \in(0,1)$, every solution $z$ of $L z=\lambda N z$ is such that $z \notin \delta \Omega$;

(b) $Q N z \neq 0$ for each $z \in \delta \Omega \cap \operatorname{Ker} L$, and the Brouwer degree $\operatorname{deg}\{J Q N, \delta \Omega \cap \operatorname{Ker} L, 0\} \neq 0$.

Then the operator equation $L z=N z$ has at least one solution in $\operatorname{Dom} L \cap \delta \Omega$. 
Definition 2 ([24]) A subset $A \subset C_{r d}(X, \mathbb{R})$ is said to be rd-equicontinuous if the following items are satisfied:

- For all right dense points of $t_{0} \in X$ and for each $\epsilon>0$, there exists $\delta>0$ such that for all $t_{0} \in \mathbb{T}$, we have

$$
\left|f(t)-f\left(t_{0}\right)\right|<\epsilon \quad \text { for all } f \in A,\left|t-t_{0}\right|<\delta
$$

- For all left dense points of $t_{0} \in X$, there exists a $\delta$-neighborhood $U_{l d}$ of $t_{0}$ such that

$$
\left|f\left(t^{\prime}\right)-f\left(t^{\prime \prime}\right)\right|<\epsilon \quad \text { for all } f \in A,\left|t^{\prime}-t^{\prime \prime}\right|<\delta \text {. }
$$

The above definition is significant for the explanation of the following Arzela-Ascoli theorem for time scales.

Theorem 2 ([24], Arzela-Ascoli theorem for time scales) Suppose that $A$ is a subset of $C_{r d}(X, \mathbb{R})$ where $X$ is the compact subspace of $\mathbb{T}$, and that the following items are satisfied:

- $A$ is uniformly bounded subset of $C_{r d}(X, \mathbb{R})$.

- $A$ is $r$-equicontinuous in $X$.

Then $A$ is a relatively compact subset of $C_{r d}(X, \mathbb{R})$.

We also give the following lemma, which is essential for the proof of the consequent theorems.

Lemma $1([4])$ Let $\tau_{1}, \tau_{2} \in[0, \omega]$ and $t \in \mathbb{T}$. If $f: \mathbb{T} \rightarrow \mathbb{R}$ is $\omega$-periodic, then

$$
f(t) \leq f\left(\tau_{1}\right)+\int_{0}^{\omega}\left|f^{\Delta}(s)\right| \Delta s \quad \text { and } \quad f(t) \geq f\left(\tau_{2}\right)-\int_{0}^{\omega}\left|f^{\Delta}(s)\right| \Delta s
$$

Remark 1 In [25] predator-prey dynamic models with several types of functional responses with impulses on time scales are studied and a general result is obtained. On the other hand, in their study, only the effect of functional response is seen on the prey, but the effect of the given functional response cannot be seen on predator. Therefore, our results are also important since the impact of Beddington-DeAngelis-type functional response is taken into account for both prey and predator.

\section{Main result}

\subsection{General case}

Remark $2([4])$ Let $\mathbb{T}=\mathbb{R}$. In (2), by taking $\exp (x(t))=\tilde{x}(t)$ and $\exp (y(t))=\tilde{y}(t)$, we obtain equality (1), which is the standard predator-prey system with Beddington-DeAngelis functional response governed by ordinary differential equations. Many studies have been done on this system, and $[1,2,14]$ are their examples.

Let $\mathbb{T}=\mathbb{Z}$. Using equality (2), we obtain

$$
\begin{aligned}
& x(t+1)-x(t)=a(t)-b(t) \exp (x(t))-\frac{c(t) \exp (y(t))}{\alpha(t)+\beta(t) \exp (x(t))+m(t) \exp (y(t))}, \\
& y(t+1)-y(t)=-d(t)+\frac{f(t) \exp (x(t))}{\alpha(t)+\beta(t) \exp (x(t))+m(t) \exp (y(t))} .
\end{aligned}
$$


Here again by taking $\exp (x(t))=\tilde{x}(t)$ and $\exp (y(t))=\tilde{y}(t)$ we obtain

$$
\begin{aligned}
& \tilde{x}(t+1)=\tilde{x}(t) \exp \left[a(t)-b(t) \tilde{x}(t)-\frac{c(t) \tilde{y}(t)}{\alpha(t)+\beta(t) \tilde{x}(t)+m(t) \tilde{y}(t)}\right], \\
& \tilde{y}(t+1)=\tilde{y}(t) \exp \left[-d(t)+\frac{f(t) \tilde{x}(t)}{\alpha(t)+\beta(t) \tilde{x}(t)+m(t) \tilde{y}(t)}\right],
\end{aligned}
$$

which is the discrete-time predator-prey system with Beddington-DeAngelis-type functional response and also the discrete analogue of (1). This system was studied in [12, 26], and [27]. Since (2) incorporates (1) and (3) as special cases, we call (2) the predator-prey dynamic system with Beddington-DeAngelis functional response on time scales.

For equation (2), $\exp (x(t))$ and $\exp (y(t))$ denote the densities of the prey and predator. Therefore, $x(t)$ and $y(t)$ may be negative. By taking the exponentials of $x(t)$ and $y(t)$ we obtain the numbers of preys and predators that are living per unit of an area. In other words, for the general time-scale case, our equation is based on the natural logarithm of the densities of the predator and prey. Hence, $x(t)$ and $y(t)$ may be negative.

For equations (1) and (3), since $\exp (x(t))=\tilde{x}(t)$ and $\exp (y(t))=\tilde{y}(t)$, the given dynamic systems directly depend on the densities of the prey and predator.

Definition 3 In system (2), if for all solutions of $x(t)(y(t)), \exp (x(t))(\exp (y(t)))$ tends to 0 as $t$ tends to infinity, then we say that the prey (predator) goes to extinction.

Lemma 2 If

$$
\int_{0}^{w}\left(-d(t)+\frac{f(t)}{\beta(t)}\right) \Delta t<0,
$$

then for all solutions of $y(t), \exp (y(t))$ tends to 0 as t tends to infinity.

Proof Using the second equation of (2), we obtain

$$
\exp (y(t)) \leq \exp (y(0)) \exp \left(\int_{0}^{t}\left(-d(t)+\frac{f(t)}{\beta(t)}\right) \Delta s\right) .
$$

Since, $\int_{0}^{w}\left(-d(t)+\frac{f(t)}{\beta(t)}\right) \Delta t<0, \lim _{t \rightarrow \infty} \exp (y(t))=0$.

Lemma 3 If the predator does not go to extinction, then neither prey does. In other words, iffor all solutions of $y(t), \exp (y(t))$ does not tend to zero as $t$ tends to infinity, then for all solutions of $x(t), \exp (x(t))$ does not tend to zero as t tends to infinity.

Proof The statement of the lemma is equivalent the statement that if the prey goes to extinction, then the predator also goes to extinction. Using the second equation in system (2) and taking the integral of that equation from 0 to $t$, we obtain

$$
\exp (y(t))=\exp (y(0)) \exp \left(\int_{0}^{t}-d(s)+\frac{f(s) \exp (x(s))}{\alpha(s)+\beta(s) \exp (x(s))+m(s) \exp (y(s))} \Delta s\right) .
$$

If the prey goes to extinction, then $\exp (x(t))$ tends to 0 as $t$ tends to infinity. Since all the coefficient functions are positive, $\frac{f(t) \exp (x(t))}{\alpha(t)+\beta(t) \exp (x(t))+m(t) \exp (y(t))}$ also tends to 0 as $t$ tends to infinity. For sufficiently large $t$, the integral $\int_{0}^{t}\left(-d(t)+\frac{f(t) \exp (x(t))}{\alpha(t)+\beta(t) \exp (x(t))+m(t) \exp (y(t))}\right) \Delta s$ becomes 
negative, and the right-hand side of the equation (4) tends to 0 as $t$ tends to infinity, which means that $\exp (y(t))$ tends to 0 as $t$ tends to infinity causing the predator to go to extinction. Hence, the proof follows.

Theorem 3 Assume that all the coefficient functions in system (2) are bounded, positive, and w-periodic, and from $C_{r d}\left(\mathbb{T}, \mathbb{R}^{2}\right)$. Then at least one w-periodic solution exists if and only if the predator does not go to extinction.

Proof Let $X:=\left\{\left[\begin{array}{l}u \\ v\end{array}\right] \in C_{r d}\left(\mathbb{T}, \mathbb{R}^{2}\right): u(t+w)=u(t), v(t+w)=v(t)\right\}$ with the norm $\left\|\left[\begin{array}{l}u \\ v\end{array}\right]\right\|=$ $\sup _{t \in[0, w] \mathbb{T}}(|u(t)|,|v(t)|)$ and $Y:=\left\{\left[\begin{array}{l}u \\ v\end{array}\right] \in C_{r d}\left(\mathbb{T}, \mathbb{R}^{2}\right), u(t+w)=u(t), v(t+w)=v(t)\right\}$ with the norm $\left\|\left[\begin{array}{c}u \\ v\end{array}\right]\right\|=\sup _{t \in[0, w]]_{T}}(|u(t)|,|v(t)|)$.

In this subsection, from now on, instead of $[0, w]_{\mathbb{T}}$, we use $[0, w]$.

Let us define the mappings $L: \operatorname{Dom} L \subset X \rightarrow Y$ and $N: X \rightarrow Y$ by

$$
L\left(\left[\begin{array}{l}
u \\
v
\end{array}\right]\right)=\left(\left[\begin{array}{l}
u^{\Delta} \\
v^{\Delta}
\end{array}\right]\right)
$$

and

$$
N\left(\left[\begin{array}{l}
u \\
v
\end{array}\right]\right)=\left(\left[\begin{array}{c}
a(t)-b(t) \exp (u(t))-\frac{c(t) \exp (v(t))}{\alpha(t)+\beta(t) \exp (u(t))+m(t) \exp (v(t))} \\
-d(t)+\frac{f(t) \exp (u(t)))}{\alpha(t)+\beta(t) \exp (u(t))+m(t) \exp (v(t))}
\end{array}\right]\right) .
$$

Then $\operatorname{Ker} L=\left\{\left[\begin{array}{l}u \\ v\end{array}\right]:\left[\begin{array}{l}u \\ v\end{array}\right]=\left[\begin{array}{c}c_{1} \\ c_{2}\end{array}\right]\right\}$, where $c_{1}$ and $c_{2}$ are constants, and

$$
\operatorname{Im} L=\left\{\left[\begin{array}{l}
u \\
v
\end{array}\right]:\left[\begin{array}{l}
\int_{0}^{w} u(s) \Delta s \\
\int_{0}^{w} v(s) \Delta s
\end{array}\right]=\left[\begin{array}{l}
0 \\
0
\end{array}\right]\right\}
$$

The set $\operatorname{Im} L$ is closed in $Y$, and $\operatorname{dim} \operatorname{Ker} L=\operatorname{codim} \operatorname{Im} L=2$. We now show that as follows. It is obvious that sum of any elements from $\operatorname{Im} L$ and $\operatorname{Ker} L$ is in $Y$. Without loss of generalization, take $u \in Y$ and $\int_{\kappa}^{w+\kappa} u(t) \Delta t=I \neq 0$. Let us define the new function $g=u-\frac{I}{\operatorname{mes}(w)}$, where $\operatorname{mes}(t)=\int_{\kappa}^{\kappa+t} 1 \Delta t$. Then $\frac{I}{\operatorname{mes}(w)}$ is constant because for all $\kappa, \int_{\kappa}^{w+\kappa} u(t) \Delta t$ is always the same by the definition of periodic time scales. Taking the integral of $g$ from $\kappa$ to $w+\kappa$, we get

$$
\int_{\kappa}^{w+\kappa} g(t) \Delta t=\int_{\kappa}^{w+\kappa} u(t) \Delta t-I=0
$$

Then $u \in Y$ can be written as the sum of $g \in \operatorname{Im} L$ and $\frac{I}{\operatorname{mes}(w)} \in \operatorname{Ker} L$, since $\frac{I}{\operatorname{mes}(w)}$ is constant. Similar steps are used for $v$. Then $\left[\begin{array}{l}u \\ v\end{array}\right] \in Y$ can be written as the sum of an element from $\operatorname{Im} L$ and an element from $\operatorname{Ker} L$. Also, it is easy to show that any element in $Y$ is uniquely expressed as the sum of an element $\operatorname{Ker} L$ and an element from $\operatorname{Im} L$. So $\operatorname{codim} \operatorname{Im} L$ is also 2 , and we get the desired result. Therefore, $L$ is a Fredholm mapping of index zero.

There exist continuous projectors $P: X \rightarrow X$ and $Q: Y \rightarrow Y$ such that

$$
P\left(\left[\begin{array}{l}
u \\
v
\end{array}\right]\right)=\frac{1}{\operatorname{mes}(w)}\left[\begin{array}{l}
\int_{0}^{w} u(s) \Delta s \\
\int_{0}^{w} v(s) \Delta s
\end{array}\right]
$$


and

$$
Q\left(\left[\begin{array}{l}
u \\
v
\end{array}\right]\right)=\frac{1}{\operatorname{mes}(w)}\left(\left[\begin{array}{l}
\int_{0}^{w} u(s) \Delta s \\
\int_{0}^{w} v(s) \Delta s
\end{array}\right]\right) .
$$

The generalized inverse $K_{P}=\operatorname{Im} L \rightarrow \operatorname{Dom} L \cap \operatorname{Ker} P$ is given by

$$
K_{P}\left(\left[\begin{array}{c}
u \\
v
\end{array}\right]\right)=\left[\begin{array}{l}
\int_{0}^{t} u(s) \Delta s-\frac{1}{\operatorname{mes}(w)} \int_{0}^{w} \int_{0}^{t} u(s) \Delta s \Delta t \\
\int_{0}^{t} v(s) \Delta s-\frac{1}{\operatorname{mes}(w)} \int_{0}^{w} \int_{0}^{t} v(s) \Delta s \Delta t
\end{array}\right],
$$

and

$$
Q N\left(\left[\begin{array}{l}
u \\
v
\end{array}\right]\right)=\frac{1}{\operatorname{mes}(w)}\left(\left[\begin{array}{c}
\int_{0}^{w}\left(a(s)-b(s) \exp (u(s))-\frac{c(s) \exp (v(s))}{\alpha(s)+\beta(s) \exp (u(s))+m(s) \exp (v(s))}\right) \Delta s \\
\int_{0}^{w}\left(-d(s)+\frac{f(s) \exp (u(s))}{\alpha(s)+\beta(s) \exp (u(s))+m(s) \exp (v(s))} \Delta s\right.
\end{array}\right]\right) .
$$

Let

$$
\begin{aligned}
& a(t)-b(t) \exp (u(t))-\frac{c(t) \exp (v(t))}{\alpha(t)+\beta(t) \exp (u(t))+m(t) \exp (v(t))}=N_{1}, \\
& -d(t)+\frac{f(t) \exp (u(t))}{\alpha(t)+\beta(t) \exp (u(t))+m(t) \exp (v(t))}=N_{2}, \\
& \frac{1}{\operatorname{mes}(w)} \int_{0}^{w}\left(a(s)-b(s) \exp (u(s))-\frac{c(s) \exp (v(s))}{\alpha(s)+\beta(s) \exp (u(s))+m(s) \exp (v(s))}\right) \Delta s=\bar{N}_{1}, \\
& \frac{1}{\operatorname{mes}(w)} \int_{0}^{w}\left(-d(s)+\frac{f(s) \exp (u(s))}{\alpha(s)+\beta(s) \exp (u(s))+m(s) \exp (v(s))}\right) \Delta s=\bar{N}_{2}, \\
& \begin{aligned}
K_{P}(I-Q) N\left(\left[\begin{array}{l}
u \\
v
\end{array}\right]\right) & =K_{P}\left(\left[\begin{array}{l}
N_{1}-\bar{N}_{1} \\
N_{2}-\bar{N}_{2}
\end{array}\right]\right) \\
& =\left[\begin{array}{l}
\int_{0}^{t}\left(N_{1}(s)-\bar{N}_{1}(s)\right) \Delta s-\frac{1}{\operatorname{mes}(w)} \int_{0}^{w} \int_{0}^{t}\left(N_{1}(s)-\bar{N}_{1}(s)\right) \Delta s \Delta t \\
\int_{0}^{t}\left(N_{2}(s)-\bar{N}_{2}(s)\right) \Delta s-\frac{1}{\operatorname{mes}(w)} \int_{0}^{w} \int_{0}^{t}\left(N_{2}(s)-\bar{N}_{2}(s)\right) \Delta s \Delta t
\end{array}\right] .
\end{aligned}
\end{aligned}
$$

Clearly, $Q N$ and $K_{P}(I-Q) N$ are continuous. Since $X$ and $Y$ are Banach spaces and they only contain the periodic functions, we can use the Arzela-Ascoli theorem for time scales and find that $\overline{K_{P}(I-Q) N(\bar{\Omega})}$ is compact for any open bounded set $\Omega \subset X$. Additionally, $Q N(\bar{\Omega})$ is bounded. Thus, $N$ is $L$-compact on $\bar{\Omega}$ for any open bounded set $\Omega \subset X$.

To apply the continuation theorem, we investigate the operator equation

$$
\begin{aligned}
& x^{\Delta}(t)=\lambda\left[a(t)-b(t) \exp (x(t))-\frac{c(t) \exp (y(t))}{\alpha(t)+\beta(t) \exp (x(t))+m(t) \exp (y(t))}\right], \\
& y^{\Delta}(t)=\lambda\left[-d(t)+\frac{f(t) \exp (x(t))}{\alpha(t)+\beta(t) \exp (x(t))+m(t) \exp (y(t))}\right] .
\end{aligned}
$$

Let $\left[\begin{array}{l}u \\ v\end{array}\right] \in X$ be a solution of system (5). Integrating both sides of system (5) over the interval $[0, w]$, we obtain

$$
\left\{\begin{array}{l}
\int_{0}^{w} a(t) \Delta t=\int_{0}^{w}\left(b(t) \exp (x(t))+\frac{c(t) \exp (y(t))}{\alpha(t)+\beta(t) \exp (x(t))+m(t) \exp (y(t))}\right) \Delta t, \\
\int_{0}^{w} d(t) \Delta t=\int_{0}^{w}\left(\frac{f(t) \exp (x(t))}{\alpha(t)+\beta(t) \exp (x(t))+m(t) \exp (y(t))}\right) \Delta t
\end{array}\right.
$$




$$
\begin{aligned}
& \int_{0}^{w}\left|x^{\Delta}(t)\right| \Delta t \\
& \quad \leq \lambda\left[\int_{0}^{w}|a(t)| \Delta t+\int_{0}^{w}\left(b(t) \exp (x(t))+\frac{c(t) \exp (y(t))}{\alpha(t)+\beta(t) \exp (x(t))+m(t) \exp (y(t))}\right) \Delta t\right] \\
& \quad \leq \lambda\left[2 \int_{0}^{w} a(t) \Delta t\right] \\
& \quad \leq M_{1}
\end{aligned}
$$

where $M_{1}:=2 \int_{0}^{w} a(t) \Delta t$,

$$
\begin{aligned}
\int_{0}^{w}\left|y^{\Delta}(t)\right| \Delta t & \leq \lambda\left[\int_{0}^{w}|d(t)| \Delta t+\int_{0}^{w} \frac{f(t) \exp (x(t))}{\alpha(t)+\beta(t) \exp (x(t))+m(t) \exp (y(t))} \Delta t\right] \\
& \leq \lambda\left[2 \int_{0}^{w} d(t) \Delta t\right] \\
& \leq M_{2}
\end{aligned}
$$

where $M_{2}:=2 \int_{0}^{w} d(t) \Delta t$.

By Theorem 1.65 from [21], since $\left[\begin{array}{l}x \\ y\end{array}\right] \in X$, there exist $\eta_{i}, \xi_{i}, i=1,2$, such that

$$
\begin{aligned}
& x\left(\xi_{1}\right)=\inf _{t \in[0, w]} x(t), \\
& x\left(\eta_{1}\right)=\sup _{t \in[0, w]} x(t), \\
& y\left(\xi_{2}\right)=\inf _{t \in[0, w]} y(t), \\
& y\left(\eta_{2}\right)=\sup _{t \in[0, w]} y(t) .
\end{aligned}
$$

By the first equation of (6) and by (7) we get $x\left(\xi_{1}\right)<l_{1}$, where $l_{1}:=\ln \left(\frac{\int_{0}^{w} a(t) \Delta t}{\int_{0}^{w} b(t) \Delta t}\right)$. Since $x\left(\xi_{1}\right)$ is the infimum of $x(t)$ for $t \in[0, w]$, there exists $t_{1} \in[0, w]$ such that $x\left(\xi_{1}\right) \leq x\left(t_{1}\right)<l_{1}$.

Using the first inequality in Lemma 1 , we have

$$
\begin{aligned}
x(t) & \leq x\left(t_{1}\right)+\int_{0}^{w}\left|x^{\Delta}(t)\right| \Delta t \leq x\left(t_{1}\right)+\left(2 \int_{0}^{w} a(t) \Delta t\right) \\
& <H_{1}:=l_{1}+M_{1} .
\end{aligned}
$$

From the second equation of (6) we have $x\left(\eta_{1}\right)>l_{2}$, where $l_{2}:=\ln \left(\frac{\int_{0}^{w} d(t) \Delta t}{\int_{0}^{w}(f(t) / \alpha(t)) \Delta t}\right)$. Since $x\left(\eta_{1}\right)$ is the supremum of $x(t)$ for $t \in[0, w]$, there exists $t_{2} \in[0, w]$ such that $x\left(\eta_{1}\right) \geq x\left(t_{2}\right)>$ $l_{2}$. By the second inequality in Lemma 1 we have

$$
\begin{aligned}
x(t) & \geq x\left(\eta_{1}\right)-\int_{0}^{w}\left|x^{\Delta}(t)\right| \Delta t \\
& \geq x\left(\eta_{1}\right)-\left(2 \int_{0}^{w} a(t) \Delta t\right) \\
& >H_{2}:=l_{2}-M_{1} .
\end{aligned}
$$


By using inequalities (11) and (12), we get $\sup _{t \in[0, w]}|x(t)| \leq B_{1}:=\max \left\{\left|H_{1}\right|,\left|H_{2}\right|\right\}$. We can write $\frac{f(t) \exp (x(t))}{\alpha(t)+\beta(t) \exp (x(t))+m(t) \exp (y(t))}$ as $\frac{f(t) \exp (y(t))}{\alpha(t)+\beta(t) \exp (x(t))+m(t) \exp (y(t))} \exp (x(t)-y(t))$. Therefore, we get

$$
\begin{aligned}
\int_{0}^{w} d(t) \Delta t & <\int_{0}^{w}(f(t) / m(t))[\exp (x(t)-y(t))] \Delta t \\
& <\left[\exp \left(x\left(\eta_{1}\right)-y\left(\xi_{2}\right)\right)\right] \int_{0}^{w}(f(t) / m(t)) \Delta t .
\end{aligned}
$$

Since (11) is true for each $t \in[0, w]$, we obtain

$$
y\left(\xi_{2}\right)<H_{1}-\ln \left(\frac{\int_{0}^{w} d(t) \Delta t}{\int_{0}^{w}(f(t) / m(t)) \Delta t}\right):=l_{3}
$$

Here $y\left(\xi_{2}\right)$ is the infimum of $y(t)$ for $t \in[0, w]$; therefore, there exists $t_{3} \in[0, w]$ such that $y\left(\xi_{2}\right) \leq y\left(t_{3}\right)<l_{3}$. Using the first equation of Lemma 1 , we have

$$
\begin{aligned}
y(t) & \leq y\left(t_{3}\right)+\int_{0}^{w}\left|y^{\Delta}(t)\right| \Delta t \\
& \leq y\left(t_{3}\right)+\left(2 \int_{0}^{w} d(t) \Delta t\right) \\
& <H_{3}:=l_{3}+M_{2} .
\end{aligned}
$$

Since all the coefficient functions in $\frac{f(t) \exp (y(t))}{\alpha(t)+\beta(t) \exp (x(t))+m(t) \exp (y(t))}$ are positive and the predator does not go to extinction, we have

$$
\frac{f(t)}{m(t)}>\frac{f(t) \exp (y(t))}{\alpha(t)+\beta(t) \exp (x(t))+m(t) \exp (y(t))}>0 .
$$

Then, there exists $k \in \mathbb{N}$ such that

$$
\frac{f(t) \exp (y(t))}{\alpha(t)+\beta(t) \exp (x(t))+m(t) \exp (y(t))}>\frac{1}{k} \frac{f(t)}{m(t)}>0
$$

and thus

$$
\begin{aligned}
\int_{0}^{w} d(t) \Delta t & =\int_{0}^{w} \frac{f(t) \exp (y(t))}{\alpha(t)+\beta(t) \exp (x(t))+m(t) \exp (y(t))}[\exp (x(t)-y(t))] \Delta t \\
& \geq\left[\exp \left(x\left(\xi_{1}\right)-y\left(\eta_{2}\right)\right)\right] \int_{0}^{w} \frac{f(t) \exp (y(t))}{\alpha(t)+\beta(t) \exp (x(t))+m(t) \exp (y(t))} \Delta t \\
& >\left[\exp \left(x\left(\xi_{1}\right)-y\left(\eta_{2}\right)\right)\right] \frac{1}{k} \int_{0}^{w} \frac{f(t)}{m(t)} \Delta t .
\end{aligned}
$$

Then we obtain

$$
y\left(\eta_{2}\right)>x\left(\xi_{1}\right)-\ln \left(\frac{\int_{0}^{w} d(t) \Delta t}{1 / k \frac{f(t)}{m(t)}}\right) .
$$


By (12) we have

$$
y\left(\eta_{2}\right)>H_{2}-\ln \left(\frac{\int_{0}^{w} d(t) \Delta t}{1 / k \frac{f(t)}{m(t)}}\right):=l_{4} .
$$

Since $y\left(\eta_{2}\right)$ is the supremum of $y(t)$ for $t \in[0, w]$, there exists $t_{4} \in[0, w]$ such that $y\left(\eta_{2}\right) \geq$ $y\left(t_{4}\right)>l_{4}$. Using the second inequality of Lemma 1 , we get

$$
\begin{aligned}
y(t) & \geq y\left(t_{4}\right)-\int_{0}^{w}\left|x^{\Delta}(t)\right| \Delta t \geq y\left(t_{4}\right)-\left(2 \int_{0}^{w} d(t) \Delta t\right) \\
> & H_{4}:=l_{4}-M_{2} .
\end{aligned}
$$

By (13) and (14) we obtain $\sup _{t \in[0, w]}|y(t)| \leq B_{2}:=\max \left\{\left|H_{3}\right|,\left|H_{4}\right|\right\}$. Obviously, $B_{1}$ and $B_{2}$ are both independent of $\lambda$. Let $M=B_{1}+B_{2}+1$. Then $\max _{t \in[0, w]}\left\|\left[\begin{array}{l}x \\ y\end{array}\right]\right\|<M$. Let $\Omega=\left\{\left[\begin{array}{l}x \\ y\end{array}\right] \in\right.$ $\left.X:\left\|\left[\begin{array}{l}x \\ y\end{array}\right]\right\|<M\right\}$, which satisfies condition (a) in Theorem 1. If $\left[\begin{array}{l}x \\ y\end{array}\right] \in \operatorname{Ker} L \cap \partial \Omega,\left[\begin{array}{l}x \\ y\end{array}\right]$ is a constant with $\left\|\left[\begin{array}{l}x \\ y\end{array}\right]\right\|=M$, then

$$
Q N\left(\left[\begin{array}{l}
x \\
y
\end{array}\right]\right)\left(\left[\begin{array}{c}
\int_{0}^{w}\left(a(s)-b(s) \exp (x)-\frac{c(s) \exp (y)}{\alpha(s)+\beta(s) \exp (x)+m(s) \exp (y)}\right) \Delta s \\
\int_{0}^{w}\left(-d(s)+\frac{f(s) \exp (x)}{\alpha(s)+\beta(s) \exp (x)+m(s) \exp (y)}\right) \Delta s
\end{array}\right]\right) .
$$

Here we take the operator $J: \operatorname{Im} V \rightarrow \operatorname{Ker} L$ as the identity operator. Then, we define the homotopy $H_{v}=v(J Q N)+(1-v) G$, where

$$
G\left(\left[\begin{array}{l}
x \\
y
\end{array}\right]\right)=\left[\begin{array}{c}
\int_{0}^{w}(a(s)-b(s) \exp (x)) \Delta s \\
\int_{0}^{w}\left(d(s)-\frac{f(s) \exp (x)}{\alpha(s)+\beta(s) \exp (x)+m(s) \exp (y)}\right) \Delta s
\end{array}\right]
$$

Take $D J_{G}$ as the determinant of the Jacobian of $G$. Since $\left[\begin{array}{l}x \\ y\end{array}\right] \in \operatorname{Ker} L$, the Jacobian of $G$ is

$$
\left[\begin{array}{cc}
-e^{x} \int_{0}^{w} b(s) \Delta s & 0 \\
\int_{0}^{w} \frac{-e^{x} f(s)}{\alpha(s)+\beta(s) e^{x}+m(s) e^{y}} \Delta s+\int_{0}^{w} \frac{\left(e^{x}\right)^{2} f(s) \beta(s)}{\left(\alpha(s)+\beta(s) e^{x}+m(s) e^{y}\right)^{2}} \Delta s & -\int_{0}^{w} \frac{e^{x} e^{y} f(s) m(s)}{\left(\alpha(s)+\beta(s) e^{x}+m(s) e^{y}\right)^{2}} \Delta s
\end{array}\right]
$$

Since all the functions in the Jacobian of $G$ are positive, $\operatorname{sign} D J_{G}$ is always positive. Hence,

$$
\operatorname{deg}(J N, \Omega \cap \operatorname{Ker} L, 0)=\operatorname{deg}(G, \Omega \cap \operatorname{Ker} L, 0)=\sum_{\left[\begin{array}{l}
x \\
y
\end{array}\right] \in G^{-1}\left(\left[\begin{array}{l}
0 \\
0
\end{array}\right]\right)} \operatorname{sign} D J_{G}\left(\left[\begin{array}{l}
x \\
y
\end{array}\right]\right) \neq 0 .
$$

Thus, all the conditions of Theorem 1 are satisfied. Therefore, system (2) has at least one positive $w$-periodic solution.

If the given system (2) has at least one periodic solution, then for all the solutions of $y(t)$, $\exp (y(t))$ does not go to zero as $t$ goes to infinity, which means that the predator does not go to extinction. Hence, we are done.

Remark 3 It is obvious that if system (2) has at least one periodic solution, then the inequality $\int_{0}^{w} d(t) \Delta t<\int_{0}^{w} \frac{f(t)}{\beta(t)} \Delta t$ must be satisfied. For the continuous case, this was done 

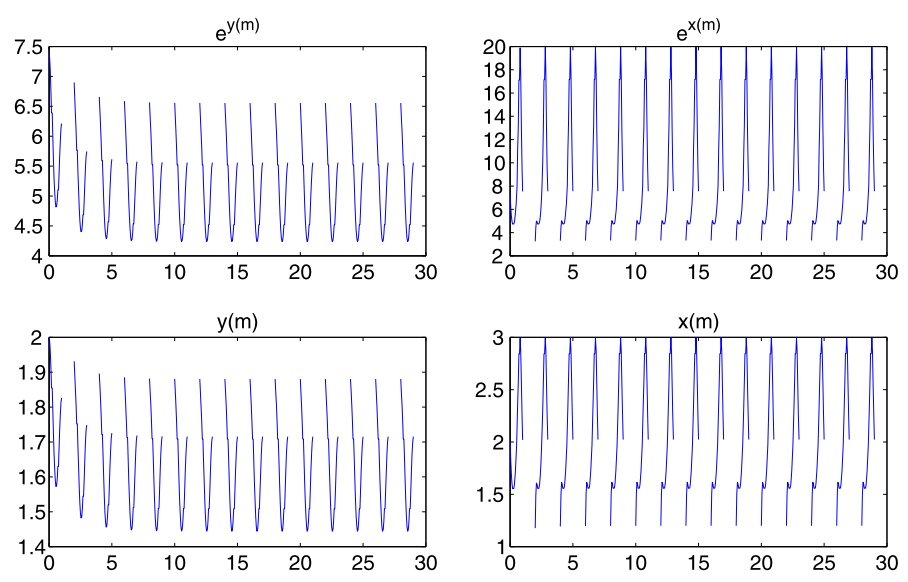

Figure 1 To obtain this figure, we take $x(0)=2$ and $y(0)=2$.

in [2]. But although this inequality is satisfied, system (2) does not have any periodic solution, which means that the predator can go to extinction by Theorem 3 . Therefore, if we are able to extend the conditions that make the predator go to extinction, then we have more information about the systems that have at least one periodic solution.

Example 1 Let $\mathbb{T}=[2 k, 2 k+1], k \in \mathbb{N}, k$ starting with 0 ,

$$
\begin{aligned}
x^{\Delta}(t)= & (2 \sin (2 \pi t)+15)-(1.5 \sin (2 \pi t)+2) \exp (x) \\
& -\frac{(4+2 \cos (2 \pi t)) \exp (y)}{(\sin (2 \pi t)+2)+(4+0.5 \cos (2 \pi t)) \exp (x)+2 \exp (y)}, \\
y^{\Delta}(t)= & -(0.7 \sin (2 \pi t)+2)+\frac{(4 \cos (2 \pi t)+10.5) \exp (x)}{(\sin (2 \pi t)+2)+(4+0.5 \cos (2 \pi t)) \exp (x)+2 \exp (y)} .
\end{aligned}
$$

Example 2 Let $\mathbb{T}=[2 k, 2 k+1], k \in \mathbb{N}_{0}$,

$$
\begin{aligned}
x^{\Delta}(t)= & (2 \sin (2 \pi t)+40)-(1.5 \sin (2 \pi t)+2) \exp (x) \\
& -\frac{(4+2 \cos (2 \pi t)) \exp (y)}{(\sin (2 \pi t)+2)+(4+0.5 \cos (2 \pi t)) \exp (x)+2 \exp (y)} \\
y^{\Delta}(t)= & -(0.7 \sin (2 \pi t)+20)+\frac{(4 \cos (2 \pi t)+100.5) \exp (x)}{(\sin (2 \pi t)+2)+(4+0.5 \cos (2 \pi t)) \exp (x)+2 \exp (y)} .
\end{aligned}
$$

Figures 1 and 2 satisfy the results obtained in Theorem 3.

\subsection{Continuous case}

\subsubsection{Preliminaries for continuous case}

Definition 4 ([28]) Solutions of a $w$-periodic system generate a $w$-periodic semiflow $T(t): X \rightarrow X(X$ is the initial value space) in the sense that $T(t) x$ is continuous in $(t, x) \in$ $[0,+\infty) \times X, T(0)=I$, and $T(t+w)=T(t) T(w)$ for all $t>0$. 

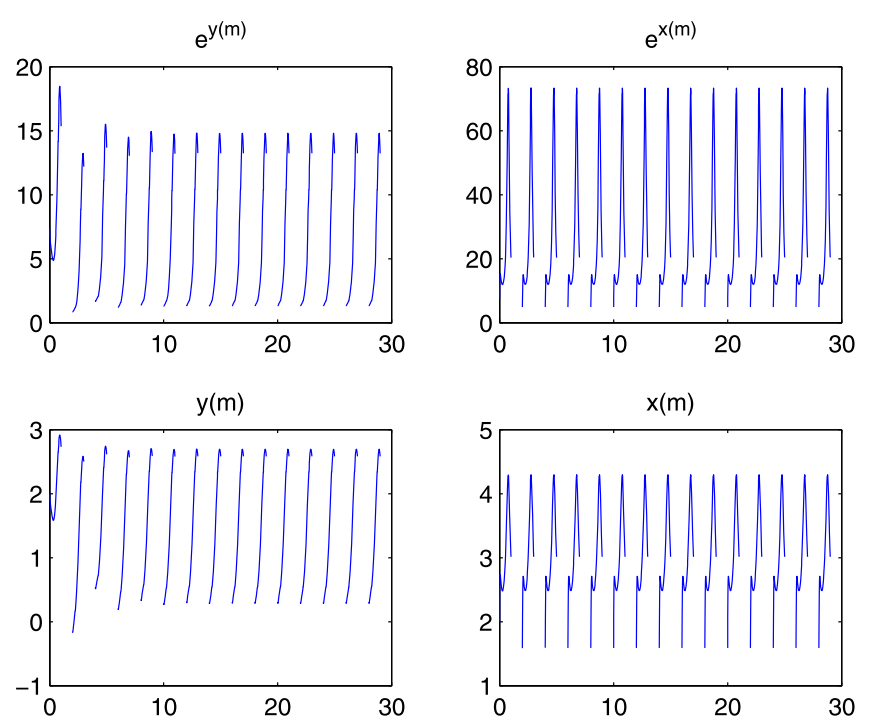

Figure 2 To obtain this figure, we take $x(0)=2$ and $y(0)=2$.

Definition 5 ([28]) The periodic semi-flow $T(t)$ is said to be uniformly persistent with respect to $\left(X_{0}, \partial X_{0}\right)$ if there exists $\eta>0$ such that for any $x \in X_{0}, \liminf _{t \rightarrow \infty} d(T(t) x$, $\left.\partial X_{0}\right) \geq \eta$.

Definition 6 ([29]) Let $T: \mathbb{R}^{n} \rightarrow \mathbb{R}^{n}$. The map $T$ is point dissipative if there exists a bounded set $B$ such that, for each $x \in \mathbb{R}^{n}$, there is an integer $n_{0}=n_{0}(x, B)$ such that $T^{n}(x) \in B$ for each $n \geq n_{0}$.

Lemma 4 ([28]) Let $S: X \rightarrow X$ be a continuous map with $S\left(X_{0}\right) \subset X_{0}$. Assume that $S$ is point dissipative, compact, and uniformly persistent with respect to $\left(X_{0}, \delta X_{0}\right)$. Then there exists a global attractor $A_{0}$ for $S$ in $X_{0}$ relative to strongly bounded sets in $X_{0}$, and $S$ has a coexistence state $x_{0} \in A_{0}$.

Definition 7 ([12]) System (1) is called permanent if there exist positive constants $r_{1}, r_{2}$, $R_{1}$, and $R_{2}$ such that solution $(\tilde{x}(t), \tilde{y}(t))$ of system (1) satisfies

$$
\begin{aligned}
& r_{1} \leq \lim _{t \rightarrow \infty} \inf \tilde{x}(t) \leq \lim _{t \rightarrow \infty} \sup \tilde{x}(t) \leq R_{1}, \\
& r_{2} \leq \lim _{t \rightarrow \infty} \inf \tilde{y}(t) \leq \lim _{t \rightarrow \infty} \sup \tilde{y}(t) \leq R_{2} .
\end{aligned}
$$

Theorem 4 ([1]) Assume that all the coefficient functions in system (1) are positive. Then system (1) is permanent and has at least one positive w-periodic solution if

$$
\frac{1}{w} \int_{0}^{w} a(t) d t\left(-d(t)+\frac{f(t) x^{*}(t)}{\alpha(t)+\beta x^{*}(t)}\right)>0,
$$

where $x^{*}(t)=\frac{1-\exp \left(-\int_{0}^{w} a(s) d s\right)}{\int_{0}^{w} b(t-s) \exp \left(-\int_{0}^{s} a(t-\tau) d \tau\right) d s}$ is the unique global asymptotically stable periodic solution of system $(1) \tilde{x}^{\prime}(t)=\tilde{x}(t)(a(t)-b(t) \tilde{x}(t))$.

In [1], the following corollary of Theorem 4 is given. 
Corollary 1 ([1]) Assume that all the coefficient functions in system (1) are positive. Then this system is permanent and has at least one w-periodic solution if

$$
\left(f^{L}-d^{M} \beta^{M}\right)\left(\frac{a}{b}\right)^{L}>d^{M} \alpha^{M}
$$

where $h^{M}$ is the maximum of $h$, and $h^{L}$ is the minimum of $h$.

Theorem 5 ([1]) Assume that all the coefficient functions in system (1) are positive. Then system (1) is permanent if and only if inequality (15) holds.

If we take $\tilde{x}=\exp (x(t))$ and $\tilde{y}=\exp (y(t))$, then the following system is equivalent to system(1):

$$
\begin{aligned}
& x^{\prime}(t)=a(t)-b(t) \exp (x(t))-\frac{c(t) \exp (y(t))}{\alpha(t)+\beta(t) \exp (x(t))+m(t) \exp (y(t))}, \\
& y^{\prime}(t)=-d(t)+\frac{f(t) \exp (x(t))}{\alpha(t)+\beta(t) \exp (x(t))+m(t) \exp (y(t))} .
\end{aligned}
$$

Definition 8 In system (17), for all solutions of $x(t)(y(t))$, if $\exp (x(t))(\exp (y(t)))$ tends to 0 as $\mathrm{t}$ tends to infinity, then we say that the prey (predator) goes to extinction. In other words, in system (1), if $\tilde{x}(t)(\tilde{y}(t))$ tends to 0 as $t$ tends to infinity, then we say that the prey (predator) goes to extinction.

In [1], a sufficient and necessary condition for the permanence of system (1) is established by the theorem, which Theorem 5 in this study. Additionally, in the discussion part of that paper, the following corollary is stated.

Corollary 2 ([1]) System (1) goes to extinction if and only if

$$
\frac{1}{w} \int_{0}^{w} a(t) d t\left(-d(t)+\frac{f(t) x^{*}(t)}{\alpha(t)+\beta x^{*}(t)}\right) \leq 0 .
$$

\subsubsection{Application of the main result to the continuous case}

Theorem 6 Assume that all the coefficient functions in system (1) are bounded, positive, $w$-periodic, and from $C\left(\mathbb{T}, \mathbb{R}^{2}\right)$. Then, there exists at least one $w$-periodic solution of system (1) if and only if inequality (15) is satisfied.

Proof First, let us assume that inequality (15) is satisfied. Then system (1) becomes permanent by Theorem 5, and the predator does not go to extinction. Since system (1) and system (17) are equivalent, the predator does not go to extinction in system (17). Then, by Theorem 3 we obtain that system (17) has at least one $w$-periodic solution. Therefore, system (1) also has at least one $w$-periodic solution.

For the other part, let us assume that our system (1) has at least one $w$-periodic solution. Then system (17) has at least one $w$-periodic solution. By Theorem 3 the predator does not go to extinction. By Lemma 3 the prey also does not go to extinction. Then $\tilde{x}(t)$ and $\tilde{y}(t)$ do not go to 0 as $t$ tends to infinity. Then by Corollary 2 we obtain that if system (1) does not go to extinction, then inequality (15) is satisfied. Hence, we are done. 
The following lemma is similar to Lemma 4.4 in [3], but with zero impulses.

Lemma 5 Suppose that inequality (15) holds. Then, the w-periodic solution of system (1) is globally asymptotically stable or globally attractive.

Proof To get the result, we apply Lemma 4. Let us consider the following ordinary differential equation:

$$
\begin{aligned}
& z^{\prime}(t)=F(t, z(t)), \\
& z(0)=\phi .
\end{aligned}
$$

Here $F \in C\left([0, \infty) \times \mathbb{R}^{2}, \mathbb{R}^{2}\right), \phi \in \mathbb{R}^{2}, F(t+w, u)=F(t, u)$. Then, the operator that solves system (18) can be written as

$$
T(t) z=z e^{-\lambda t}+\int_{0}^{t} e^{-\lambda(t-s)}[F(s, T(s) z)+\lambda T(s) z] d s,
$$

where $\lambda$ is a positive constant. It is obvious that $T(0)=I$. Also, we can verify that

$$
u(s)= \begin{cases}T(s) z, & 0 \leq s \leq w \\ T(s-w) T(w) z, & w \leq s \leq t+w\end{cases}
$$

is the solution of system (18) with the initial value $u(0)=z$, where $s \in[0, t+w]$. By the uniqueness theorem system (18) has a unique solution; therefore, $T(t+w) z=u(t+w)=$ $T(t) T(w) z$.

To apply Lemma 4 , let $S=T(w), S^{2}=S \circ S=T(w) \circ T(w)=T(2 w)$. Here the considered system (18) is a periodic system. Therefore, we can apply Theorem 2 and obtain that $T(t)$ is a compact operator. Additionally, the compactness of the given operator can also be shown by the following alternative way. In [3], Theorem 4.4, the considered system is

$$
\begin{aligned}
& z^{\prime}(t)=F(t, z(t)), \\
& z\left(t_{k}^{+}\right)-z\left(t_{k}\right)=I_{k}\left(z\left(t_{k}\right)\right), \\
& z(0)=\phi .
\end{aligned}
$$

Here $I_{k} \in C\left(\mathbb{R}^{2}, \mathbb{R}^{2}\right)$, and the solution operator of system (19) is defined as

$$
\hat{T}(t) z=z e^{-\lambda t}+\int_{0}^{t} e^{-\lambda(t-s)}[F(s, \hat{T}(s) z)+\lambda \hat{T}(s) z] d s+\sum_{0<t_{k}<t} e^{-\lambda\left(t-t_{k}\right)} I_{k}\left(\hat{T}\left(t_{k}\right) z\right) .
$$

If we take $I_{k}$ as the zero function, then system (19) is becomes system (18), and the solution operator $\hat{T}$ becomes equal to the solution operator $T$. By [3] and [30], $T(t)$ is a compact operator. Then $S$ is a compact operator. If we take $X_{i}^{+}=\left\{z_{i}: z_{i} \in \mathbb{R}, z_{i} \geq 0\right\}$ for $i=1,2$ and $X_{i_{0}}^{+}=\left\{z_{i}: z_{i} \in \mathbb{R}, z_{i}>0\right\}$ for $i=1,2$, then $X=X_{1}^{+} \times X_{2}^{+}, X=X_{1_{0}}^{+} \times X_{2_{0}}^{+}$, and $\delta X_{0}=X / X_{0}$. When system (1) satisfies inequality (15), system (1) becomes permanent. Therefore, $S$ satisfies the conditions of Lemma 4 . Therefore, $S$ admits a global attractor, which means that the system has a globally asymptotically stable or globally attractive $w$-periodic solution. 
Corollary 3 Assume that all the coefficient functions in system (1) are bounded, positive, w-periodic, and from $C\left(\mathbb{T}, \mathbb{R}^{2}\right)$. Then, there a globally attractive w-periodic solution for system (1) exists if and only if inequality (15) is satisfied.

Proof Proof is immediate from Lemma 5 and Theorem 6.

\subsubsection{Examples for continuous case}

Example 3 Consider

$$
\begin{aligned}
x^{\prime}(t)= & (2 \sin (2 \pi t)+3)-(0.2 \sin (2 \pi t)+0.4) \exp (x) \\
& -\frac{(5+2 \cos (2 \pi t)) \exp (y)}{(\sin (2 \pi t)+1.2)+(1+0.5 \sin (2 \pi t)) \exp (x)+\exp (y)}, \\
y^{\prime}(t)= & -(0.5 \sin (2 \pi t)+1.5)+\frac{(0.8 \cos (2 \pi t)+4.45) \exp (x)}{(\sin (2 \pi t)+1.2)+(1+0.5 \sin (2 \pi t)) \exp (x)+\exp (y)} .
\end{aligned}
$$

By some calculations we obtain that $x^{*} \geq \frac{5 \exp \left(-\frac{\cos (2 \pi t)}{\ln }\right)}{\exp (1 / \pi)}:=x^{* *}$. Then we get

$$
\begin{gathered}
-(0.5 \sin (2 \pi t)+1.5)+\frac{(0.8 \cos (2 \pi t)+4.45) x^{*}(t)}{(\sin (2 \pi t)+1.2)+(1+0.5 \sin (2 \pi t)) x^{*}(t)} \\
>-(0.5 \sin (2 \pi t)+1.5)+\frac{(0.8 \cos (2 \pi t)+4.45) x^{* *}(t)}{(\sin (2 \pi t)+1.2)+(1+0.5 \sin (2 \pi t)) x^{* *}(t)}>0 .
\end{gathered}
$$

This means that Example 3 satisfies inequality (15) and has a globally attractive 1-periodic solution. Figure 3 also supports our findings.

Although we change the initial values of the system in Example 3, we get the same solution after a while as it is seen in Figure 4. This shows the global attractivity of the 1-periodic solution of Example 3.
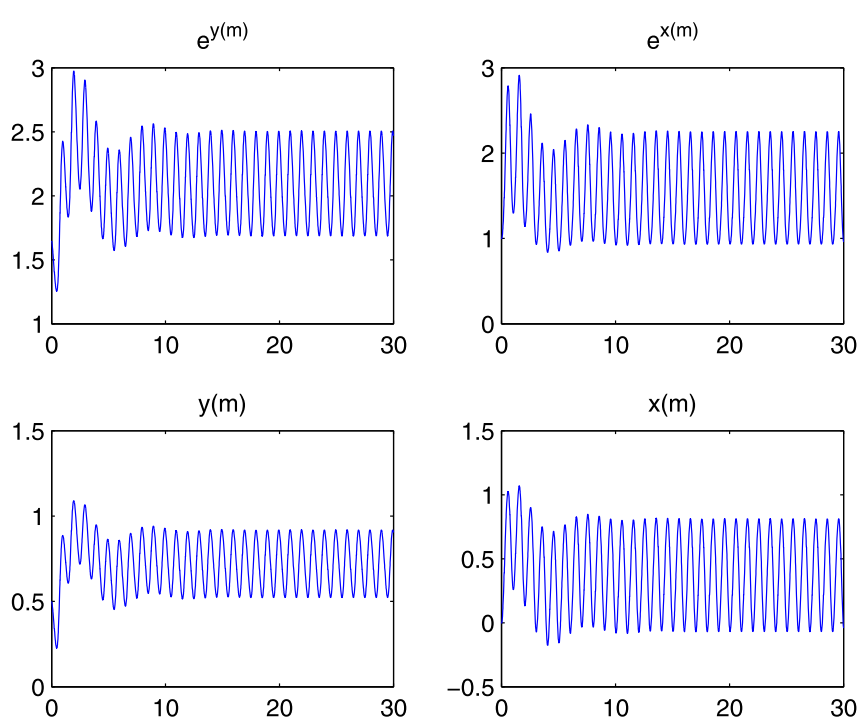

Figure 3 To obtain this figure, we take $x(0)=0.1$ and $y(0)=0.5$. 

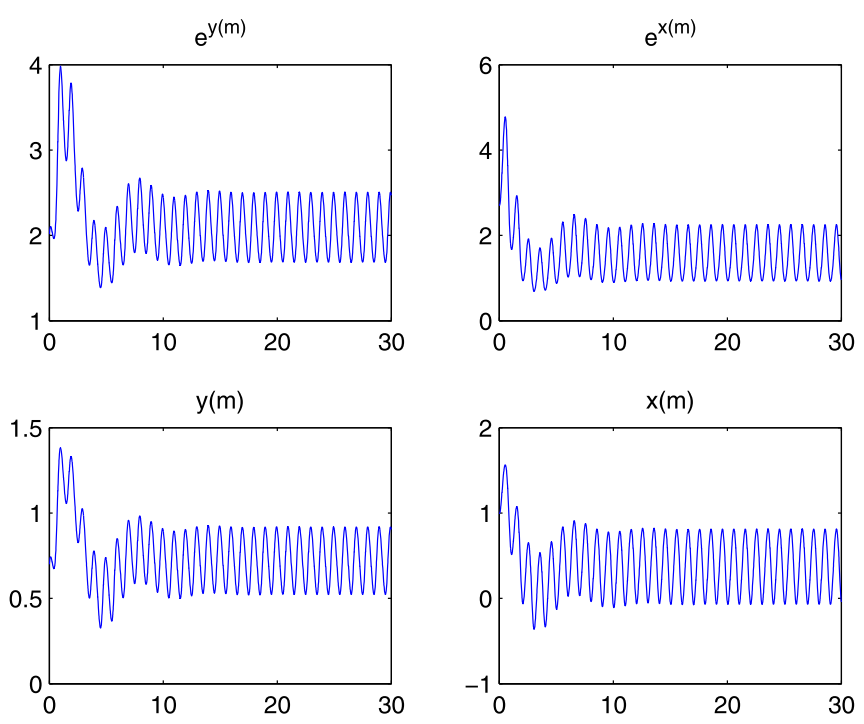

Figure 4 To obtain this figure, we take $x(0)=1$ and $y(0)=0.7$.

For Example 3, $\left(f^{L}-d^{M} \beta^{M}\right)\left(\frac{a}{b}\right)^{L}=(3.65-(2) \cdot(1.5)) \cdot(5)=3.25$ and $d^{M} \alpha^{M}=2 \cdot(2.2)=$ 4.4 , but $4.4>3.25$. Therefore, this example does not satisfy inequality (16) in Corollary 1 , but since it satisfies inequality (15), we can say that this system has a 1-periodic globally attractive solution.

Example 4 Consider

$$
\begin{aligned}
x^{\prime}(t)= & (\sin (2 \pi t)+3)-(0.5 \sin (2 \pi t)+7) \exp (x) \\
& -\frac{(10+\cos (2 \pi t)) \exp (y)}{(0.1 \sin (2 \pi t)+10)+\exp (x)+5 \exp (y)}, \\
y^{\prime}(t)= & -(0.5 \sin (2 \pi t)+1.2)+\frac{(\cos (2 \pi t)+10) \exp (x)}{(0.1 \sin (2 \pi t)+10)+\exp (x)+5 \exp (y)} .
\end{aligned}
$$

Here the inequality is as follows:

$$
\int_{0}^{w}-d(t)+\frac{f(t)}{\beta(t)} d t=\int_{0}^{1}-(0.5 \sin (2 \pi t)+1.2)+(\cos (2 \pi t)+10) d t=8.8>0 .
$$

According to the study of [2], if the inequality were $\int_{0}^{w}-d(t)+\frac{f(t)}{\beta(t)} d t<0$, then we could obtain the result that system goes to extinction. However, since we find that this inequality is greater than zero, we cannot make any observation about whether the system goes to extinction or not. For this reason, we use inequality (15). After some calculations we obtain that $x^{*} \leq 0.6$. Then we get

$$
\begin{aligned}
& -(0.5 \sin (2 \pi t)+1.2)+\frac{(\cos (2 \pi t)+10) x^{*}(t)}{(0.1 \sin (2 \pi t)+10)+x^{*}(t)} \\
& \leq-(0.5 \sin (2 \pi t)+1.2)+\frac{(\cos (2 \pi t)+10) 0.6}{(0.1 \sin (2 \pi t)+10)+0.6} \\
& <-0.08<0 .
\end{aligned}
$$



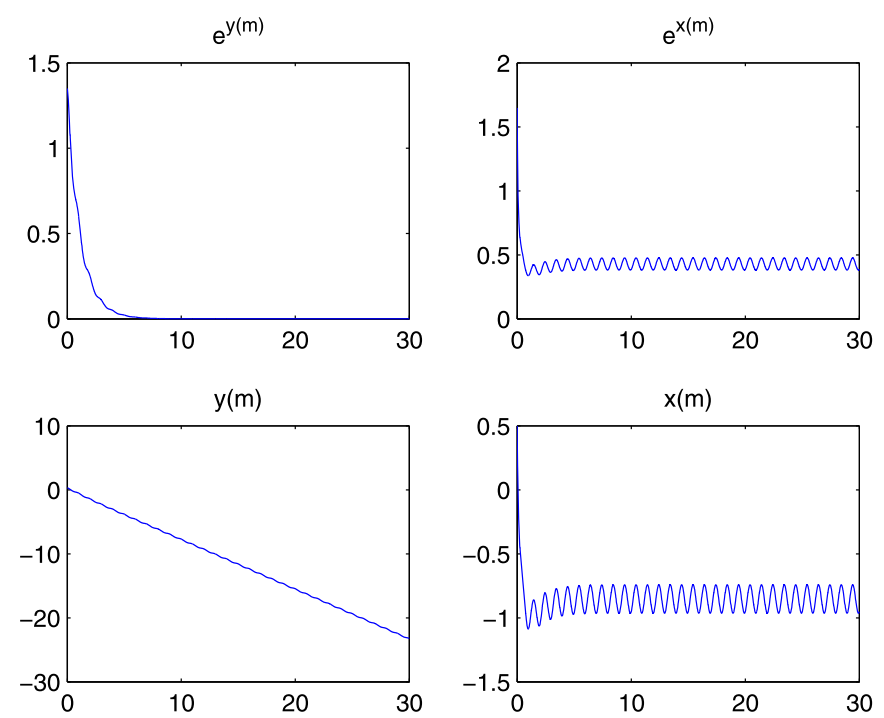

Figure 5 To obtain this figure we take $x(0)=0.5$ and $y(0)=0.3$.

Since Example 4 does not satisfy inequality (15), the system does not have a periodic solution, the predator of this system goes to extinction, and Figure 5 satisfies this result.

All of the figures in this study are obtained by Matlab program.

\section{Discussion}

In [2], Figure 1, for the continuous case, there was a discussion about why Figure (1a) does not satisfy the conditions of Theorem 3.2 in that paper, but the solutions are still periodic. We can answer this question by using our Theorem 3 . When the coefficient functions in Figure (1a) are bounded, positive, 1-periodic, continuous, and the predator does not go to extinction, then we have at least one 1-periodic solution, which is globally asymptotically stable. Inequality (15) was first found by Cui and Takeuchi [1]. However, what they have found was the equivalence between satisfaction of inequality (15) and the permanence of the predator-prey dynamic systems with Beddington-DeAngelis-type function response. Although they have found that if system (1) satisfies inequality (15), then it has at least one $w$-periodic solution, they could not say anything about when system (1) has at least one $w$-periodic solution, whether it satisfies inequality (15) or not. In that paper, by using Theorem 3 and Theorem 6 , we can say that for system (1), having at least one $w$-periodic solution of is equivalent to satisfaction of inequality (15), which means that a much better development of the inequality for system (1) to investigate the periodic solution is impossible. In addition, by using Corollary 3 we are able to say that satisfaction of inequality (15) is equivalent to the existence of a globally attractive $w$-periodic solution.

Hence, for any continuous predator-prey dynamic system with Beddington-DeAngelistype functional response, there is a globally attractive $w$-periodic solution if and only if inequality (15) is satisfied, and the predator of this system goes to extinction if and only if inequality (15) is not satisfied.

As a result, the importance of this paper is that we can enlarge conditions for the existence of the positive periodic solutions of predator-prey dynamic systems with 


\title{
Beddington-DeAngelis functional response for continuous case and for a general time- scale case.
}

\author{
Competing interests \\ The authors declare that they have no competing interests.
}

\section{Authors' contributions}

NNP gave the first idea of studying necessary and sufficient conditions of the considered system and also made the literature review. Additionally, NNP desinged the pattern of the study, and all findings were controlled by AFG in each step with BK. NNP participated in the contribution in the mathematical analysis part, and his contributions were controlled by AFG and BK. All of the authors drafted the manuscript together. BK and AFG gave the final approval. All authors read and approved the final manuscript.

\section{Author details}

'Department of Mathematics, Ondokuz Mayıs University, Samsun, Turkey. ${ }^{2}$ Department of Mathematics, Faculty of Science, Ankara University, Ankara, 06590, Turkey. ${ }^{3}$ Department of Mathematics and Computer Science, Çankaya University, Ankara, 06810, Turkey.

\section{Acknowledgements}

We thank the reviewers and the academic editor of this article for all their contributions in the review process.

\section{Received: 30 July 2015 Accepted: 10 January 2016 Published online: 22 January 2016}

\section{References}

1. Cui, J, Takeuchi, Y: Permanence, extinction and periodic solution of predator-prey system with Beddington-DeAngelis functional response. J. Math. Anal. Appl. 317, 464-474 (2006)

2. Fan, $M$, Kuang, Y: Dynamics of a nonautonomous predator-prey system with the Beddington-DeAngelis functional response. J. Math. Anal. Appl. 295, 15-39 (2004)

3. Wang, W, Shen, J, Nieto, J: Permanence and periodic solution of predator-prey system with Holling type functional response and impulses. Discrete Dyn. Nat. Soc. 2007, Article ID 81756 (2007)

4. Bohner, M, Fan, M, Zhang, J: Existence of periodic solutions in predator-prey and competition dynamic systems. Nonlinear Anal., Real World Appl. 7, 1193-1204 (2006)

5. Fan, M, Wang, Q: Periodic solutions of a class of nonautonomous discrete time semi-ratio-dependent predator-prey systems. Discrete Contin. Dyn. Syst., Ser. B 4(3), 563-574 (2004)

6. Beddington, JR: Mutual interference between parasites or predators and its effect on searching efficiency. J. Anim. Ecol. 44, 331-340 (1975)

7. DeAngelis, DL, Goldstein, RA, O'Neill, RV: A model for trophic interaction. Ecology 56, 881-892 (1975)

8. Chen, F: Permanence and global stability of nonautonomous Lotka-Volterra system with predator-prey and deviating arguments. Appl. Math. Comput. 173, 1082-1100 (2006)

9. Fan, M, Agarwal, S: Periodic solutions for a class of discrete time competition systems. Nonlinear Stud. 9(3), 249-261 (2002)

10. Fan, M, Wang, K: Global periodic solutions of a generalized $n$-species Gilpin-Ayala competition model. Comput. Math. Appl. 40(10-11), 1141-1151 (2000)

11. Fan, M, Wang, K: Periodicity in a delayed ratio-dependent predator-prey system. J. Math. Anal. Appl. 262(1), 179-190 (2001)

12. Fang, Q, Li, X, Cao, M: Dynamics of a discrete predator-prey system with Beddington-DeAngelis function response. Appl. Math. 3, 389-394 (2012)

13. Huo, HF: Periodic solutions for a semi-ratio-dependent predator-prey system with functional responses. Appl. Math. Lett. 18, 313-320 (2005)

14. Li, H, Takeuchi, Y: Dynamics of density dependent predator-prey system with Beddington-DeAngelis functional response. J. Math. Anal. Appl. 374, 644-654 (2011)

15. Wang, Q, Fan, M, Wang, K: Dynamics of a class of nonautonomous semi-ratio-dependent predator-prey systems with functional responses. J. Math. Anal. Appl. 278(2), 443-471 (2003)

16. Xu, R, Chaplain, MAJ, Davidson, FA: Periodic solutions for a predator-prey model with Holling-type functional response and time delays. Appl. Math. Comput. 161(2), 637-654 (2005)

17. Hilger, S: Analysis on measure chains - a unified approach to continuous and discrete calculus. Results Math. 18 18-56 (1990)

18. Fazly, M, Hesaaraki, M: Periodic solutions for predator-prey systems with Beddington-DeAngelis functional response on time scales. Nonlinear Anal., Real World Appl. 9, 1224-1235 (2008)

19. Güvenilir, AF, Kaymakçalan, B, Pelen, NN: Impulsive predator-prey dynamic systems with Beddington-DeAngelis type functional response on the unification of discrete and continuous systems. Appl. Math. 6, 1649 (2015)

20. Yang, L, Yang, J, Zhog, Q: Periodic solutions for a predator-prey model with Beddington-DeAngelis type functional response on time scales. Gen. Math. Notes 3(1), 46-54 (2011)

21. Bohner, M, Peterson, A: Dynamic Equations on Times Scales: An Introduction with Applications. Birkhäuser, Basel (2001)

22. Bourbaki, N: Elements of Mathematics. Algebra: Algebraic Structures. Linear Algebra, 1. Addison-Wesley, Reading (1974) (Chaps. 1, 2)

23. Gaines, RE, Mawhin, JL: Coincidence Degree and Non-Linear Differential Equations. Springer, Berlin (1977)

24. Gong, Y, Xiang, X: A class of optimal control problems of systems governed by the first order linear dynamic equations on time scales. J. Ind. Manag. Optim. 5(1), 1-10 (2009) 
25. Liu, X, Liu, X: Necessary and sufficient conditions for the existence of periodic solutions in a predator-prey model on time scales. Electron. J. Differ. Equ. 2012199 (2012)

26. Zhang, J, Wang, J: Periodic solutions for discrete predator-prey systems with the Beddington-DeAngelis functional response. Appl. Math. Lett. 19, 1361-1366 (2006)

27. Xu, C, Liao, M: Existence of periodic solutions in a discrete predator-prey system with Beddington-DeAngelis functional responses. Int. J. Math. Math. Sci. 2011, Article ID 970763 (2011)

28. Zhao, XQ: Uniform persistence and periodic coexistence states in infinite-dimensional periodic semiflows with applications. Can. Appl. Math. Q. 3(4), 473-495 (1995)

29. Hale, JK: Asymptotic Behavior of Dissipative Systems. Math. Surveys and Monographs, vol. 25. Am. Math. Soc., Providence (1988)

30. Bainov, D, Simeonov, P: Impulsive Differential Equations: Periodic Solutions and Applications. Pitman Monogr. Surveys Pure Appl. Math., vol. 66. Longman, Harlow (1993)

Submit your manuscript to a SpringerOpen ${ }^{\odot}$ journal and benefit from:

- Convenient online submission

Rigorous peer review

- Immediate publication on acceptance

- Open access: articles freely available online

- High visibility within the field

- Retaining the copyright to your article 